\title{
High Temperature Elastic Constants and the Evaluation of Effective Pair Potentials
}

\author{
Raymond D. Mountain* and Donald C. Knauss $†$ \\ National Bureau of Standards, Washington, D.C. 20234 \\ October 17, 1979
}

\begin{abstract}
It is shown by example that the predicted temperature dependence of the elastic constants is a useful measure of the ability of an effective pair potential to estimate the high temperature thermal properties of a metal. Our example is based on a model pair potential constructed for aluminum. This potential predicts the low temperature elastic constants and phonon dispersion relations with good accuracy ( \pm a few percent). The high temperature elastic constants for this model potential are determined using the Monte Carlo method and are found to be approximately independent of temperature. Since the elastic constants of aluminum are strongly decreasing functions of temperature, this potential is seen to be a poor one for determining the properties of aluminum. We conclude that the temperature dependence of the elastic constants is a useful further test of pair potentials which satisfy the low temperature tests currently employed.
\end{abstract}

Key words: Aluminum, anharmonic effects, effective potentials for metals, elastic constants, fluctuations, high temperature properties, Monte Carlo simulations

\section{Introduction}

Pseudopotential theory has made it possible to develop effective, volume dependent pair potentials for metals [1] ${ }^{1}$ and thereby to estimate the high temperature thermal properties of metals using the methods of statistical mechanics [2-4]. When a model pseudopotential is used, it is necessary to fit the model parameters to various physical properties such as the low temperature lattice parameter and elastic constants or dispersion curves in the harmonic approximation. [5]. Fitting an effective potential to low temperature properties is no guarantee that the high temperature properties of the solid will also be satisfactorily predicted. Cohen et al. [4] have suggested that the ability of an effective potential to predict reliably the high temperature elastic constants of a metal is a good test of the potential. In this paper we show by example that this is the case and assert that the predicted temperature dependence of the elastic constants is a useful measure of the ability of an effective potential to estimate high temperature thermal properties of a metal.

Our discussion is in terms of a model potential for aluminum which produces good dispersion curves at low temperatures but which produces totally unacceptable estimates

\footnotetext{
*Center for Thermodynamics and Molecular Science, National Measurement Laboratory. +NBS-NRC Postdoctoral Research Associate 1976-1978.

Current Address: Chemistry Department, Oregon State University, Corvallis, Oregon 97331

' Figures in brackets indicate the literature references at the end of this paper.
}

for the temperature dependence of the elastic constants at high temperatures. The elastic constants are determined at high temperatures, where anharmonic effects are important, using Monte Carlo techniques [2-4] to evaluate the fluctuation expressions of Squire, et al. [2]. While the static lattice estimates for elastic constants employ only a discrete set of values of the derivatives of the potential, the full microscopic expressions [2] sample in principle the entire range of the potential when anharmonic effects ars important. Thus the predicted temperature variation of the elastic constants for a fixed volume depends on the overall shape of the potential. This is demonstrated by our example.

This paper is organized as follows. Section 2 contains a discussion of the pair potential we have devised for aluminum. We show that it satisfies the compressibility sum rule and state that it reliably predicts the low temperature elastic constants and dispersion curves for aluminum. Section 3 describes the Monte Carlo calculation of the temperature dependence of the elastic constants at high temperatures and Section 4 summarizes our results.

\section{Pair potential}

Numerous effective pair potentials have been proposed for aluminum and the shape of the potential has been found to be quite sensitive the details of the electron screening 
function. Duesbery and Taylor have shown that a necessary condition for a potential is that the screening function satisfy the electron gas compressiblity theorem [6]. In the same vein, Hafner and Schmuck [7] have stated that this compressibility sum rule should be at least approximately satisfied if the correct relation between longitudinal and transverse branches is to be realized.

We have found empirically that if the combination of elastic constants $2 C^{\prime}-C=C_{11}-C_{12}-C_{44}$ is correctly predicted then the dispersion curves for the tranverse modes in the $(1,1,0)$ direction are correctly ordered. Here $C_{11}, C_{12}$ and $C_{44}$ are cubic elastic constants in the usual notation. [8] We examined the model pseudopotential of Dagens, et al [9] which satisfies the compressibility sum rule and found that it predicts a crossing of the dispersion curves for the transverse modes in the $(1,1,0)$ direction. We have also examined the first principles pseudopotential of Harrison [1] and found that it too predicts mode crossing. Hafner and Schmuck [7] developed a corrected Harrison potential and used it to calculate the elastic constants of aluminum at zero temperature. Their results are in agreement with the experimental values of Kamm and Ahlers [10].

The model potential used in this calculation approximately satisfies the compressibility sum rule and correctly predicts the value of the combination of elastic constants $2 C^{\prime}-C=C_{11}-C_{12}-C_{44}$ at zero temperature. Since errors which do not influence the compressibility rule may affect $2 C^{\prime}-C$, we observe that a potential which satisfies both conditions at zero temperature is more likely to function adequately at non zero temperatures than one which does not. As we shall demonstrate, this is not a sufficient condition. From pseudopotential theory we know that given the energy wave number characteristic, $F(q)$, we can obtain by Fourier transformation the indirect (electron gas induced) interaction between the ion cores. The full poten. tial is the sum of the couloumb and indirect interactions. For simplicity we have used a model $F(q)$ as recently applied to the calculation of the structure factor of liquid aluminum [11]. This model $F(q)$ introduced by Ashcroft and Langreth [12] is expressed as

$$
F(q)=\frac{4 \pi Z^{2} \mathrm{e}^{2}}{\Omega q^{2}} \cos ^{2}\left(q R_{i o n}\right)\left[\frac{1}{\varepsilon(q)}-1\right]
$$

where $Z e$ is the charge on the ion, $\Omega$ is the volume occupied by an ion, $q$ is the wave vector, $R_{\text {ion }}$ is a parameter given a value of 1.12 atomic units for aluminum and $\varepsilon(q)$ is the dielectric function given by [13]

$$
\varepsilon(q)=1+\frac{2 \mathrm{k}_{\mathrm{f}}}{\pi q^{2}}\left[\frac{R(q)}{1-\frac{2 k_{f}}{\pi q^{2}} \psi(q) R(q)}\right]
$$

where $k_{f}$ is the Fermi wave vector for a free electron at the density in question. The function $R(q)$ is defined by

$$
R(q)=\frac{1}{2}+\frac{4 k_{f}^{2}-q^{2}}{8 k_{f} q} \ln \left|\frac{2 k_{f}+q}{2 k_{f}-q}\right|
$$

and $\psi(q)$ is the exchange screening function. We have used the Hubbard model for $\psi(q)$;

$$
\psi(q)=\frac{1}{2} \frac{q^{2}}{q^{2}+\xi k_{f}^{2}}
$$

with the parameter assigned the value $\xi=1.699$ so that the compressibility sum rule is satisfied. The full potential $V(r)$ is given by

$$
V(r)=\frac{(Z e)^{2}}{r}+\frac{\Omega}{\pi^{2}} \int_{0}^{\infty} d q q^{2} F(q) \frac{\sin q r}{q r}
$$

This potential is displayed in figure $l$ as a function of $r$. It adequately reproduces the low temperature phonon dispersion relations in the harmonic approximation [14] and also avoids the crossing of the nearly degenerate small $k$ transverse modes in the $(1,1,0)$ direction. Thus it satisfies what we consider to be a minimum requirement for a "good" potential function. The local maximum located between the lst and 2nd neighbor distances is a common feature of many effective potentials for aluminum $[6,9]$.

The zero temperature (static lattice) values of $2 C^{\prime}-C$ and the lattice compressibility $\beta_{\mathrm{T}}$ can be simply expressed as

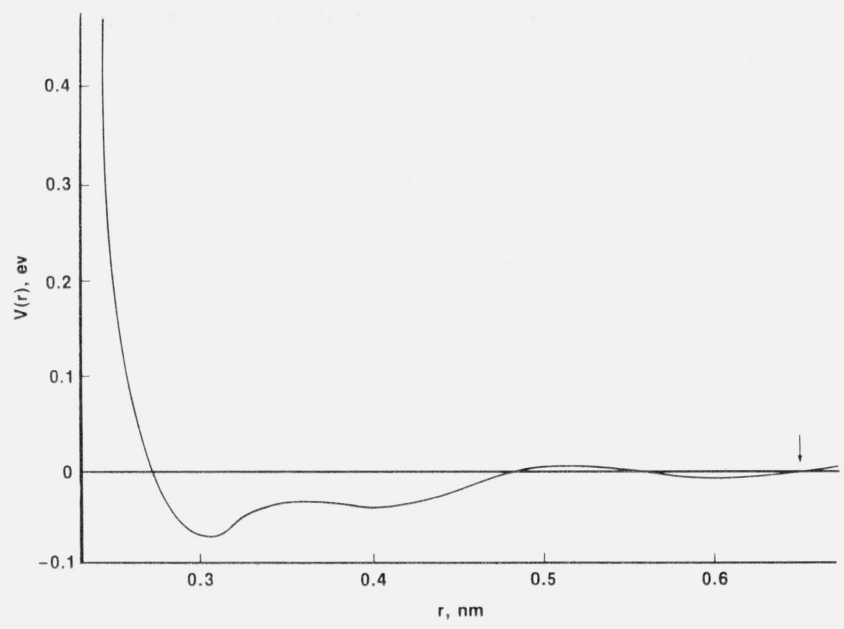

FIGURE 1. The effective pair potential for aluminum at a density of $2.718 \mathrm{~g} / \mathrm{cm}^{3}$ (lattice parameters $=.404 \mathrm{~nm}$ ).

The energy is in units of electron volts and the distance is in nanometers. The arrow indicates the place where the potential was set to zero in the Monte Carlo Calculations. 


$$
2 C^{\prime}-C=\frac{4}{a_{o}}\left\{f_{2}+\frac{16}{5} f_{5}+\ldots\right\}
$$

and

$3 / \beta_{\mathrm{T}}=C_{11}+2 C_{12}$

$$
=\frac{4}{a_{o}}\left\{f_{1}+f_{2}+3 f_{3}+4 f_{4}+10 f_{5}+\ldots\right\}
$$

where

$$
f_{n}=\left[\frac{\partial^{2} V(r)}{\partial r^{2}}-\frac{1}{r} \frac{\partial V(r)}{\partial r}\right]_{r=r_{n}}
$$

with $r_{n}$ the distance to the $n^{\text {th }}$ neighbor shell and $a_{o}$ is the lattice parameter. These expressions are obtained by summing over shells Squire et al's [2] equations for the elastic constants of the static FCC lattice. The values of the first five $f_{n}$ 's for the potential of figure 1 are listed in table 1.

TABLE I

\begin{tabular}{lc}
\hline \hline$n$ & $f_{n}-$ dynes $/ \mathrm{cm}$ \\
\hline 1 & 20.5790 \\
2 & 2.0479 \\
3 & -2.0199 \\
4 & 0.0872 \\
5 & 0.0515 \\
\hline
\end{tabular}

The predicted low temperature value of $2 C^{\prime}-C$ is obtained by using table 1 and eq (2.5) to obtain

$$
2 C^{\prime}-C=2.31 \times 10^{11} \text { dynes } / \mathrm{cm}^{2} \text {. }
$$

This is to be compared with the experimental value [15] of $2.327 \times 10^{11}$ dynes $/ \mathrm{cm}^{2}$. The calculated value for the compressibility is

$$
3 / \beta_{\mathrm{T}}=1.694 \times 10^{12} \text { dynes } / \mathrm{cm}^{2}
$$

which is to be compared with the experimental value of $1.612 \times 10^{12}$ dynes $/ \mathrm{cm}^{2}$. The lattice compressibility sum rule is satisfied to within 5 percent and the $2 C^{\prime}-C$ rule to within 1 percent by this potential.

\section{Monte Carlo calculations}

The Monte Carlo method of Metropolis, et al [3] was used to calculate the thermal properties of the crystalline model using the effective pair potential of figure 1. The system consisted of 256 particles located initially on the FCC sites of a $4 \times 4 \times 4$ block of unit cells with lattice parameter $a=$ $0.404 \mathrm{~nm}$. Periodic boundary conditions were used to mimic an infinite system. A simulation started with an "ageing" period of $3 \times 10^{5}$ trials. The data were extracted from the $1 \times 10^{6}$ trials following the ageing sequence. The program adjusted the maximum step size allowed in a trial move so that approximately $1 / 2$ of the proposed moves were accepted. Double precision arithmetic was used to accumulate the data and all other calculations employed single precision arithmetic. The use of double precision is necessary if $10^{6}$ samples are to be averaged using 32-bit words.

The temperature dependent part of the pressure was evaluated by averaging the virial. The elastic constants were obtained by evaluating the expressions developed by Squire, et al. [2] and our program was checked by reproducing their results for the Lennard-Jones solid.

Estimates were obtained for the energy per particle $(E)$, the pressure (p), the isothermal elastic constants $\left(C_{11} T, C_{12}{ }^{T}\right.$, $C_{44}{ }^{T}$ ), the difference between the isothermal and adiabatic elastic constants $(\Delta \mathrm{C})$, the specific heat at constant volume $\left(C_{\mathrm{v}}\right)$ and the derivative of the pressure with respect to temperature at constant volume $((\partial P / \partial T) v)$. Fluctuation expressions for $C_{v}$ and $(\partial P / \partial T) v$ were used to estimate these quantities [4-16].

The full expressions for the pressure, $C_{11}$ and $C_{12}$ as functions of volume and temperature contain electron gas terms which are functions of the volume, but are independent of configuration and temperature [4]. Since we are concerned only with the temperature variation of these quantities, we have not included these contributions in our results which are listed in table 2 . The quoted uncertainties are based on the stability of the estimates as the Monte Carlo process progresses. This is illustrated in figure 2 where the estimates for $\mathrm{P},(\partial P / \partial T) v$ and $C_{11}{ }^{T}$ at $500 \mathrm{~K}$ are shown as a function of the number of trials after the ageing process had been completed. The program provides these estimates every $5 \times 10^{4}$ trials. Only $8.5 \times 10^{5}$ trials were made for $T=700 \mathrm{~K}$ due to a computer malfunction which made continuation of that run impossible.

TABLE 2

\begin{tabular}{cccc}
\hline \hline $\begin{array}{c}\text { Temperature } \\
\text { (Number of samples) }\end{array}$ & $\begin{array}{c}500 \mathrm{~K} \\
\left(10^{6}\right)\end{array}$ & $\begin{array}{c}600 \mathrm{~K} \\
\left(10^{6}\right)\end{array}$ & $\begin{array}{c}700 \mathrm{~K} \\
\left(8.5 \times 10^{5}\right)\end{array}$ \\
\hline $\begin{array}{c}\text { Property } \\
E, 10^{-13} \mathrm{erg} / \text { atom } \ldots \ldots\end{array}$ & $-6.004 \pm .001$ & $-5.602 \pm .001$ & $-5.188 \pm .001$ \\
$P, 10^{11} \mathrm{dyne} / \mathrm{cm}^{2} \ldots$ & $1.643 \pm .001$ & $1.689 \pm .001$ & $1.737 \pm .001$ \\
$(\partial \mathrm{P} / \partial \mathrm{T})$, & & & \\
$10^{7} \mathrm{dyne} / \mathrm{cm}^{2} \cdot \mathrm{K} \ldots$ & $5.0 \pm .2$ & $4.8+.2$ & $4.7 \pm .2$ \\
$C_{v}, 10^{-16} \mathrm{erg} / \mathrm{K} \cdot$ atom & $4.2 \pm .2$ & $4.2 \pm .2$ & $4.1 \pm .2$ \\
$C_{11}{ }^{T}, 10^{11} \mathrm{dyne} / \mathrm{cm}^{2} \cdots$ & $6.4 \pm .3$ & $6.6 \pm .3$ & $6.7 \pm .3$ \\
$C_{12}{ }^{T}, 10^{11} \mathrm{dyne} / \mathrm{cm}^{2} \ldots$ & $0.97 \pm .3$ & $1.1 \pm .3$ & $1.1 \pm .3$ \\
$C_{44}{ }^{T}, 10^{11} \mathrm{dyne} / \mathrm{cm}^{2} \cdots$ & $1.2 \pm .2$ & $1.4 \pm .2$ & $1.4 \pm .2$ \\
$\Delta C, 10^{10} \mathrm{dyne} / \mathrm{cm}^{2} \cdots$ & $4.9 \pm .5$ & $5.6 \pm .5$ & $6.3 \pm .5$ \\
\hline
\end{tabular}




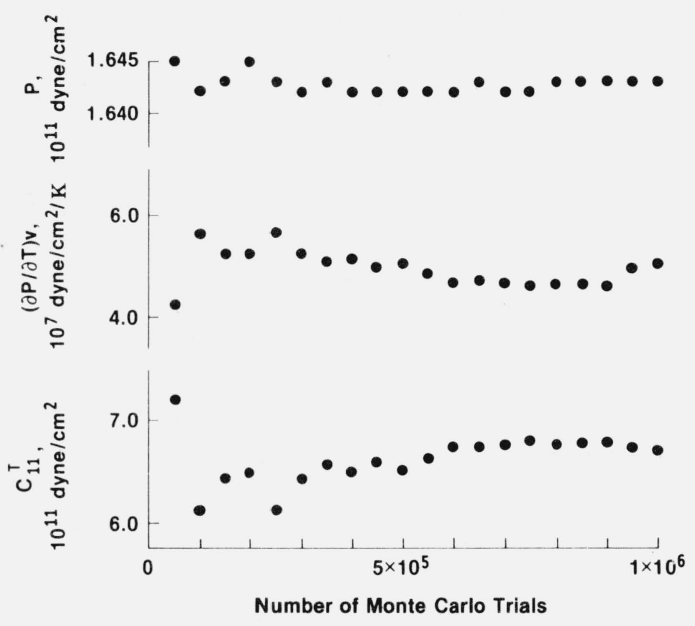

FIGURE 2. Running estimates for the pressure $(P)$, the derivative of the pressure with respect to temperature at constant volume $\left((\partial P / \partial T)_{V}\right)$ and the isothermal elastic constant $C_{11}{ }^{T}$ for the $T=500 \mathrm{~K}$ state.

Values are plotted for intervals of $5 \times 10^{4}$ trials.

The estimates for the pressure and the energy are quite stable while those for the other quantities, all of which involve fluctuations, exhibit substantial variations with the number of Monte Carlo trials. The variation shown here is substantially larger than that found for the elastic constants of sodium by Cohen et al. [4].

\section{Conclusions}

Our principal conclusion is that the temperature dependence of the elastic constants, for fixed volume, is a useful further test of pair potentials which satisfy low temperature tests. By examining the temperature dependence under fixed volume conditions, it is possible to sample the full range of the effective potential without introducing the complication of volume dependent changes in the potential itself.

The calculated temperature dependence of the elastic constants is zero within the uncertainties quoted in table 2 .
This is certainly not the case for aluminum [17] where the elastic constants are decreasing functions of temperature. We see that the potential of figure 1, which works quite nicely at low temperatures is not at all a suitable potential function for aluminum. We also observe that a good deal of care is needed to insure that the Monte Carlo averages are stable. To achieve this, fairly long (in terms of the number of trials) simulations may be necessary. Finally, we conjecture that the local maximum shown in figure 1 . between the first and second neighbor distances is responsible for the unphysical temperature dependence of the elastic constants. This conjecture is based on the idea that the maximum inhibits anharmonic effects and thereby makes the lattice "stiffer" than it should be.

\section{References}

[1] Harrison, W. A., Pseudopotentials in the Theory of Metals, (W. A. Benjamin, New York, 1965).

[2] Squire, D. R., Holt, A. C. and Hoover, W. G., Physica 42, 388 (1969).

[3] Metropolis, N., Rosenbluth, A. W., Rosenbluth, M. N., Teller, A. H. and Teller, E., J. Chem. Phys. 21, 1087 (1953).

[4] Cohen, S. S., Klein, M. L., Duesberg, M. S. and Taylor, R., J. Phys. F: Metal Phys. 6, 337, L271 (1976).

[5] Cohen, M. L. and Heine, V., Solid State Physics Vol. 24, H. Ehrenreich, F. Seitz and D. Turnbull, eds (Academic Press, New York, 1979) pp. 37-248.

[6] Duesbery, M. S. and Taylor, R., Phys. Rev. B 7, 2870 (1973).

[7] Hafner, J. and Schmuck, P., Phys. Rev. B 9, 4138 (1973).

[8] Kittel C., Introduction to Solid State Physics 2nd edition (John Wiley and Sons, Inc., New York, 1956) p. 91.

[9] Dagens, L., Rasolt, M. and Taylor, R., Phys. Rev. B11, 2726 (1975).

[10] Kamm, G. N. and Alers, G. A., J. Appl. Phys. 35, 327 (1964).

[11] Ebbsjo, I., Kinell, T. and Waller, I., J. Phys. C: Solid State 11, L501 (1978).

[12] Ashcroft, N. W. and Langreth, D. C., Phys. Rev. 155, 682 (1967).

[13] Geldart, D. J. W. and Vosko, S. H., Can. J. Phys. 44, 2137 (1966).

[14] Stedman, R. and Nilsson, G., Phys. Rev. 145, 492 (1966).

[15] Trappeniers, N. J., Biswas, S. N., Van't Klooster, P. and tenSandam, C. A., Physica B 85, 33 (1977).

[16] Cheung, P. Y., Molec. Phys. 13, 519 (1977).

[17] Gerlich, D. and Fisher, E. S., J. Phys. Chem. Solids 30, 1197 (1969).

[18] Binder, K. in Monte Carlo Methods in Statistical Physics, K. Binder, ed. (Springer-Verlag, New York, 1979) p. 42. 